\title{
Numerical Simulation Study of Aerodynamic Characteristic on Hypersonic Aircraft
}

\author{
Jiang CHEN \\ School of Aeronautic Science and Engineering, Beihang \\ University, \\ Beijing 100191, P. R. China; \\ chjig168@126.com
}

\author{
Song-ping WU \\ School of Aeronautic Science and Engineering, Beihang \\ University, \\ Beijing 100191, P. R. China; \\ National Laboratory for Computational Fluid Dynamics, \\ Beihang University, \\ Beijing 100191, P. R. China; \\ Ministry of Education Key Laboratory of Fluid Mechanics, \\ Beihang University, \\ Beijing 100191, P.R. China
}

\begin{abstract}
In this paper, a three dimension model that is similar to the hypersonic cruise aircraft $\mathrm{X}-51 \mathrm{~A}$ has been generated by using a graphics processing technology and an anti-modeling method from the published pictures and related articles about the X-51A. This paper adopts the Harten-Yee' s scheme to simulate the aircraft at the cruise state of $M_{\infty}=6, \quad \alpha=6^{\circ}$. And related results are acquired. Analyze the basic characteristics of the flow-field qualitatively and find that the result is kept well with the designed state. So it means that the model could be used for the further related research.
\end{abstract}

Key words-hypersonic; aerodynamic characteristic; numerical simulation

\section{I . INTRODUCTION ${ }^{1}$}

With the inspiratory engine or assembled engine as the main force, the aircraft that could do the medium and long distance flight in the atmosphere and past the atmosphere with the speed beyond the Mach number 5 is called the hypersonic aircraft [1]. This aircraft has been become more and more favored by many advanced countries. And developing a new type hypersonic aircraft, for example the X43-A [2] and X-51A [3], is even more the world's military power's priority.

It is different from the traditional cruise aircraft that the fuselage and the propulsion system are separate relatively. The scramjet located underneath the X-51A hypersonic aircraft is taken as its propulsion system. The forebody can provide precompression airflow for the scramjet and the expansion of the nozzle can offer extra push. The integrative property of fuselage and engine leads the hypersonic aircrafts to have a complex configuration. Looking into the public pictures and related published papers, a model that is similar to the X-51A has been generated by using the anti-model method. This paper carries out the CFD numerical simulation and elementarily analyzes its aerodynamic characteristic and related parameters in order to further study about the hypersonic aircraft.

\footnotetext{
${ }^{1}$ Project supported by the National Natural Science Foundation of China (NO. 91016006)
}

\section{II . Modeling ANd CALCUlating Meshes}

\section{A. Modeling}

Consulted related pictures and papers, a model similar to $\mathrm{X}-51 \mathrm{~A}$ has been obtained by using an anti-modeling method. In view of machining and heat protecting, the leading edge should be blunt and the blunt radius should not be exceeded its character length $1 / 100$ of the middleweight [4]. The blunt method is adopted Tincher's [5]. The model is shown in Fig.1.

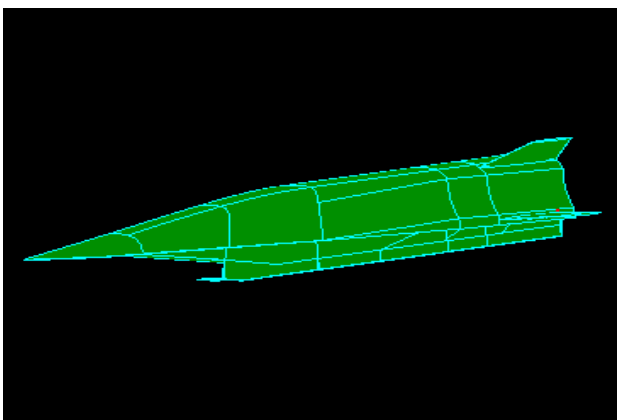

Figure 1. The model

\section{B. Calculating Meshes}

Because of its symmetry, the calculating zone is only a half of its model. The three dimension structure meshes are created by used the Gridgen software, shown in Fig.2. The flow of the boundary layer is very complicated, so the normal meshes next to the wall should be local refinements. And the distance between the first layer and the wall is $5 \times 10^{-5}$ meters.

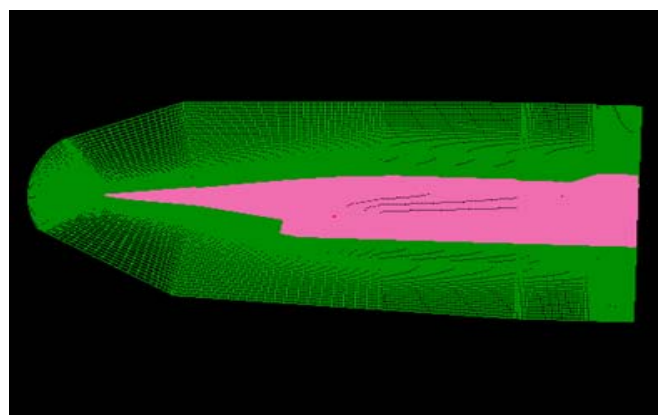

Figure 2 . The mesh 


\section{CONTROL EQUATIONS AND INITIAL CONDITIONS}

\section{A. Control equations}

In the body-fitted coordinates, the three-dimensional unsteady compressible Navier-Stokes equations can be written as follows:

$$
\frac{\partial \boldsymbol{Q}}{\partial t}+\frac{\partial \boldsymbol{F}}{\partial \xi}+\frac{\partial \boldsymbol{G}}{\partial \eta}+\frac{\partial \boldsymbol{H}}{\partial \zeta}=\frac{1}{\operatorname{Re}}\left(\frac{\partial \boldsymbol{F}_{\boldsymbol{v}}}{\partial \xi}+\frac{\partial \boldsymbol{G}_{\boldsymbol{v}}}{\partial \eta}+\frac{\partial \boldsymbol{H}_{\boldsymbol{v}}}{\partial \zeta}\right)
$$

Where $\boldsymbol{Q}$ is defined as:

$$
\mathbf{Q}=J(\rho, \rho u, \rho v, \rho w, e)
$$

$e$ is an energy per unit mass, $J$ is Jacobi coordinate transformation matrix, $\boldsymbol{F}, \boldsymbol{G}, \boldsymbol{H}$ is separately the inviscid flux of $\xi, \eta, \zeta$ direction and $\boldsymbol{F}_{v}, \boldsymbol{G}_{v}, \boldsymbol{H}_{v}$ is the viscid flux of $\xi, \eta, \zeta$ direction.

In this paper, the time discretization is adopted the LU-SGS method proposed by Yoon and Jameson and the viscid flux is adopted the second order central difference scheme. But the inviscid flux is used the Harten-Yee's second order upwind TVD scheme in order to improve the resolution of the shock wave.

\section{B. Initial Conditions}

This paper intends to simulate the aircraft's aerodynamic characteristic at the state of cruise. So the initial conditions of flow-field can be given as follows: $\rho=0.01801 \mathrm{~kg} / \mathrm{m} 3$, $\mathrm{p}=1172 \mathrm{~Pa}, \quad \mathrm{Ma} \infty=6, \mathrm{~T} \infty=226.65 \mathrm{~K}$, the wall is adopted the model of isothermal wall and $T_{\text {wall }}=294.44 \mathrm{~K}$, $\mathrm{R} \infty / \mathrm{m}=2.2 \times 10^{6}$, and with no-slip wall boundary condition.

\section{RESULTS AND ANALYSIS}

The model's forehead is adopted the wave rider. In other words, it is based on the ideal wave rider's lower surface and leading edges, and then created the upper surface so as to keep the wave rider's flow-field structure to the greatest extent. Because the wave rider realizes its wave effect mainly via the lower surface which compresses the inflow.

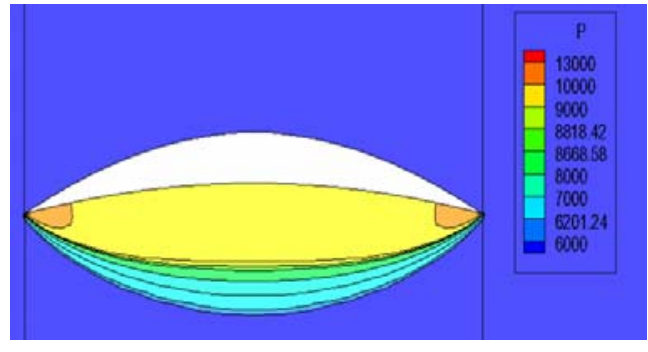

Figure 3 . Pressure contours of ideal wave-rider

Fig. 3 is the cross section pressure contour of ideal wave-rider. From the it we can clearly find out that the shock wave produced by the wave-rider's lower surface is totally separated by the lower surface, and the shocks can be able to ride on the wave-rider very well. So the strong pressure difference between upper and lower surface can offers wave rider a lot of lifts.

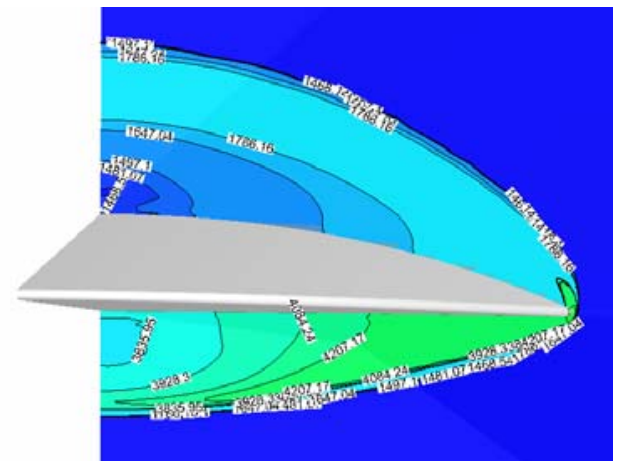

Figure 4 . Pressure contours of wave-rider with blunt edge

From the Fig.4, the pressure beneath the lower surface is obviously larger than that of upper surface. But at the edge, the lower surface and the upper surface are united together. So the high-pressure air beneath the lower surface is pulled into a low-pressure zone above the upper surface. The pressure difference between two surfaces is decreased, and so the lift. The lift-to-drag ratio is down, too.

From the table 1, we know that the blunt edge results in the lift decreasing and the drag increasing. The lift coefficient of two different types of model is decline, but not much, only $3.85 \%$. But the drag coefficient and the lift-to-drag ration changes sharply, the drag coefficient increases $215 \%$ and the lift-to-drag ration is dropped from 14.521 to 4.437 . Because the blunt leading-edge changes the flow-field structure, the airflow is stopped at the leading-edge and a detached bow shock is generated. So the characteristic of wave-rider does not exist.

TABLE I . AERODYNAMIC COEFFICIENT

\begin{tabular}{|c|c|c|c|}
\hline Model type & Lift coefficient & $\begin{array}{c}\text { Drag } \\
\text { coefficient }\end{array}$ & $\begin{array}{c}\text { Lift-to-drag } \\
\text { ration }\end{array}$ \\
\hline Ideal wave-rider & 0.1628735 & 0.0112163 & 14.521 \\
\hline $\begin{array}{c}\text { Wave-rider with blunt } \\
\text { edge }\end{array}$ & 0.1566014 & 0.0352934 & 4.437 \\
\hline
\end{tabular}

The aircraft's blunt edge will generate a bow shock when the air flows through it with the speed of Mach number $M \infty=6$ and the attack angle $\alpha=6^{\circ}$. The shock moves on and is secondly compressed at the compression surface. Then a stronger shock is generated. From the Fig.5, we can see that two ways of shock intersect well at the lip of inlet which is our expectance. The compressed air belonged to the second shock totally flows into the inlet, and its high pressure is adjusted to a stable pressure that is suit for the firebox when working in the air-inlet.

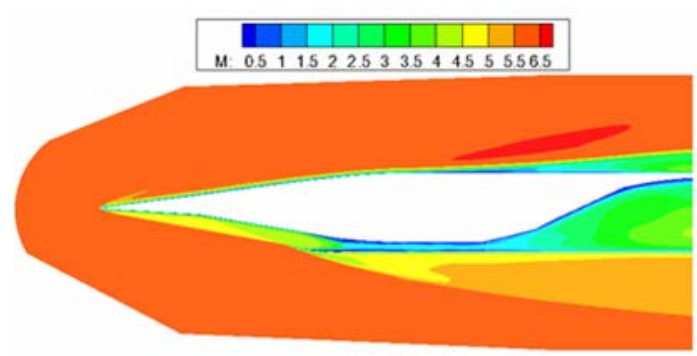

Figure 5 . Ma contours at the symmetry with $\alpha=6^{\circ}$

The velocity of airflow is sharply decreased after the air-inlet. For one thing, it is the viscidity effect that makes the boundary layer airflow's velocity not equal. So different layer's friction as well as the friction of the layer and the 
wall leads the kinetic energy cut down, and the velocity of airflow is cut down, too. For another, after the shock run into the air-inlet, it reflects at the wall many times, the pressure has been improved, but the energy is lost a lot during the process of fierce collision. It also results in the loss of velocity.

At the end of the fuselage, there is an expanded nozzle, the airflow's velocity gradually increased. From the Rawalpindi nozzle principle we know that the supersonic airflow's velocity is increased along with the cross section increased. So it also offers additional push.

\section{CONCLUSION}

This paper utilizes the anti-modeling method to obtain the model similar to X-51A with the help of published pictures and related papers, creates meshes and does the numerical calculation. At last the primary flow-filed is acquired. We can get several conclusions as follows from the analysis of flow-filed:

( i ) To some extent, the forebody with wave configuration can keep the wave-rider's character well, but as result of blunt edge, the high pressure airflow below lower surface is leaked, the lift is decreased, and so is the lift-to-drag ration.

(ii) The first shock that is created when the supersonic airflow flows through the aircraft intersects the second shock which is created by the compression surface at the lip of air inlet, making the compressed airflow belonged to the second shock entirely flow into the inlet. This can save the mechanism design that is used to compress the inlet air and improve the facility utilization.

(iii) Though the model is based on published pictures and papers, the analysis of numerical simulation indicates that the model is available for the further study.

\section{ACKNOWLEDGMENT}

R. B. G. thanks the National Natural Science Foundation of China (NO. 91016006).

\section{REFERENCE}

[1] Guo-biao Cai, Da-jun Xu, The Technology of Hypersonic Vehicle, 2012

[2] McClinton C.R., Rausch V.L., Nguyen L.T., et al. Prelimilary X-43A Flight Test Results [J]. Acta Astrpnautica, 2005, 57:266

[3] Joseph M. Hank, James S. Murphy, and Richard C. Mutzman. The X-51A Scramjet Engine Flight Demonstration Program. AIAA 2008-2540,2008

[4] Jian-hui $\mathrm{Wu}$, Song-ping $\mathrm{Wu}, 16^{\text {th }}$ National Supersonic Academic Conference of Aerodynamic and Aerodynamic Heating, 2011, 60-63

[5] Tincher D, Lane J. On the design of a hypersonic waverider test bed vehicle:a first step to outer planet exploration[R]. AIAA 92-0308, 1992. 